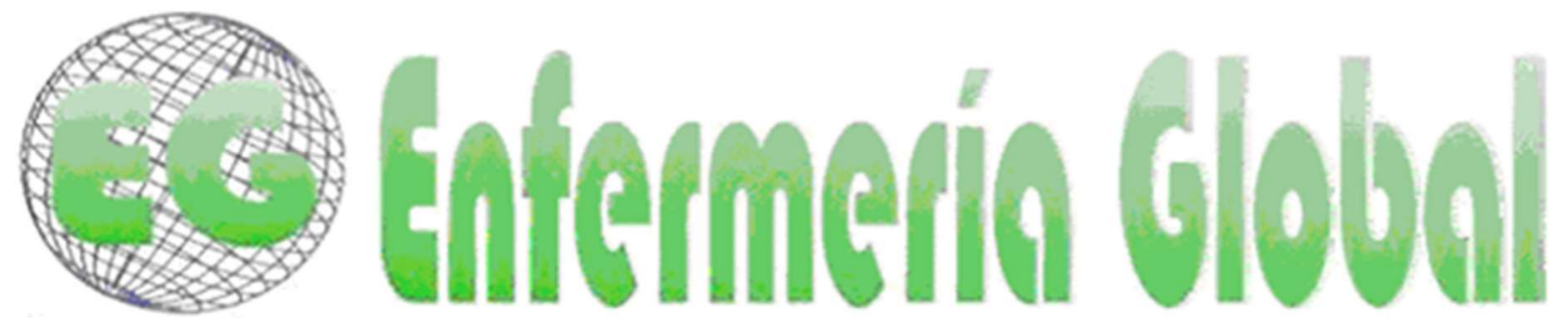

\title{
ORIGINALES
}

\section{Investigación-acción en las prácticas pedagógicas sobre la enseñanza del cuidado: Experiencia docente}

Action research in pedagogical practices of teaching caring: Teaching experience

\author{
Jessica Belen Rojas-Espinoza ${ }^{1}$ \\ Beatriz Elizabeth Martinez-Talavera ${ }^{2}$ \\ Lucila Cárdenas-Becerril ${ }^{3}$ \\ Liliana Inés Benhumea Jaramillo 4 \\ Beatriz Arana Gómez ${ }^{5}$ \\ Silvana Silveira Kempher ${ }^{6}$
}

\begin{abstract}
${ }^{1}$ Doctora en Ciencias de la Salud. Profesora e investigadora de la Facultad de Enfermería y Obstetricia, Universidad Autónoma del Estado México. Miembro del Sistema Nacional de Investigadores (SNI). México.

2 Maestra en Enfermería Quirúrgica. Profesora de la Facultad de Enfermería y Obstetricia, Universidad Autónoma del Estado de México. talavera.be@gmailcom

3 Doctora en Educación. Profesora e investigadora de la Facultad de Enfermería y Obstetricia de la Universidad Autónoma del Estado de México. Miembro del Sistema Nacional de Investigadores (SNI) nivel I. México.

4 Maestra en Enfermería en Terapia Intensiva. Profesora de la Facultad de Enfermería y Obstetricia, Universidad Autónoma del Estado de México.

5 Doctora en Enfermería. Profesora e investigadora de la Facultad de Enfermería y Obstetricia de la Universidad Autónoma del Estado de México.

6 Doctora en Enfermería. Profesora e investigadora de la Universidad Federal de Santa Catarina, Florianópolis, Brasil.
\end{abstract}

\section{https://doi.org/10.6018/eglobal.480671}

Recibido: 22/05/2021

Aceptado: 17/07/2021

\section{RESUMEN:}

Introducción: La enseñanza del cuidado requiere especial atención, así como reconocer su naturaleza representacional, cuál es su dinámica y cuáles son sus relaciones con la propia práctica.

Objetivo: Intervenir en la capacitación de profesores para la enseñanza del cuidado, mediante la interpretación y concientización de sus prácticas pedagógicas que trasciendan en el desempeño académico de los estudiantes de la Licenciatura en Enfermería.

Metodología: Investigación-acción con enfoque cualitativo-interpretativo. La intervención se llevó a cabo mediante un curso-taller sobre la enseñanza del cuidado, impartido por las investigadoras a 30 docentes de práctica y clínica en el pregrado de Enfermería. El curso se estructuró mediante la metodología del Arco de Charles Maguerez, en sus cinco etapas. Los encuentros de las sesiones fueron observados de forma participante, registrados en un diario de campo y audio-grabados, para posterior transcripción y análisis de contenido. Se conformaron categorías de análisis a partir de las reflexiones y participaciones de los docentes: a) Significado de la enseñanza del cuidado, b) Prácticas 
pedagógicas a partir del ejemplo (currículum oculto) y c) Identificación de necesidades para mejorar las prácticas pedagógicas.

Resultados: Entre los discursos de los profesores se identificó que el significado de la enseñanza del cuidado trasciende en la relación humana; que las prácticas pedagógicas tienen que promover la relación y acompañamiento al estudiante, así como el empoderamiento, la seguridad y confianza en sí mismo y que existen necesidades pedagógicas y didácticas para favorecer el aprendizaje y vincular la teoría y la práctica.

Conclusiones: Se requiere fortalecer los equipos de trabajo y la capacitación continua en la enseñanza del cuidado, distinguiendo que existen oportunidades de mejora en el desarrollo de las prácticas pedagógicas.

Palabras clave: Educación en enfermería, Cuidado, Enfermería, Enseñanza, Prácticas pedagógicas.

\begin{abstract}
:
Introduction: The teaching of care requires special attention, as well as recognizing its representational nature, what is its dynamics and what are its relationships with the practice itself.

Objective: To intervene in the training of teachers for the teaching of care, through the interpretation and awareness of their pedagogical practices that transcend the academic performance of the students of the Nursing Degree.

Methodology: Action research with a qualitative-interpretive approach. The intervention was carried out through a course workshop on the teaching of care, given by the researchers to 30 practice and clinical teachers in undergraduate Nursing. The course was structured using the Charles Maguerez Arc methodology in its five stages. The meetings of the sessions were observed in a participatory way, recorded in a field diary and audio-recorded, for later transcription and content analysis. Categories of analysis were formed from the reflections and participation of the teachers: a) Meaning of the teaching of care, b) Pedagogical practices based on the example (hidden curriculum) and, c) Identification of needs to improve pedagogical practices.

Results: Among the professors' speeches it was identified that the meaning of the teaching of care transcends in the human relationship; those pedagogical practices must promote the relationship and accompaniment of the student, as well as empowerment, security, and self-confidence, and that there are pedagogical and didactic needs to favor learning and link theory and practice.

Conclusions: It is necessary to strengthen work teams and continuous training in the teaching of care, distinguishing that there are opportunities for improvement in the development of pedagogical practices.
\end{abstract}

Keywords: Nursing education, Care, Nursing, Teaching, Pedagogical practices.

\title{
INTRODUCCIÓN
}

La enfermería en su currículo formativo ejecuta una práctica educativa hacia el cuidado de la salud, a partir de estrategias y acciones pedagógicas que propenden restaurar o mejorar las condiciones de salud de la población; por lo tanto, el cuidado debe estar centrado en una práctica pedagógica para consolidar una formación universitaria basada en el humanismo y la justicia social, de ahí que las prácticas pedagógicas constituyen un proceso que se despliega en el aula, laboratorio o campo de acción, en donde la relación docente-estudiante se centra en la construcción y aplicación del conocimiento implicando aprendizajes compartidos ${ }^{(1)}$.

En este sentido, el cuidado de enfermería envuelve y comparte la experiencia humana a través de una relación transpersonal de respeto ${ }^{(1,2)}$, confianza y diálogo entre docentes y estudiantes; dimensiones del cuidado que se aprenden a través del tiempo y que es importante practicarlo y modelarlo en la formación del profesional de enfermería, pues el docente se convierte en el modelo a seguir para el estudiante y es posible que imite ciertos comportamientos. Derivado de esto, los profesores reconocen, para enseñar el cuidado de Enfermería, es necesario hacerlo con ejemplos prácticos y anécdotas, para que puedan discutir y comprender lo que 
explican los libros o las evidencias científicas con lo que en realidad se hace en la práctica ${ }^{(3)}$.

En lo que concierne a las estrategias didácticas en la enseñanza del cuidado, los docentes deben tener en cuenta aspectos esenciales como: Los problemas de motivación hacia el aprendizaje y hacia la profesión que pueden tener los estudiantes, el medio en el que viven, el escolar y el área clínica; lo cual permitirá establecer un vínculo e identidad como futuro profesional de enfermería ${ }^{(4)}$.

Además, la innovación de estrategias de aprendizaje para la integración de la teoría y la práctica en enfermería constituyen el reto actual en la disciplina, por lo que el pensamiento crítico y la orientación teórica son herramientas que facilitan la consolidación de la identidad disciplinar y la toma de decisiones congruentes con el sistema de valores de su disciplina ${ }^{(5)}$.

De esta manera, el entorno educativo demanda en los docentes un dominio amplio disciplinar y pedagógico; deben considerar la diversidad en la enseñanza que permita que los estudiantes posean confianza, esperanza, coraje, paciencia, continuidad y constancia, además de los conocimientos disciplinarios, que en conjunto garanticen la formación de recursos humanos activos y proactivos en el cuidado de la salud en cualquier entorno.

Para la realización del presente estudio se partió de un diagnóstico obtenido mediante una investigación previa a éste, en donde se identificaron dificultades y desafíos en la praxis de las prácticas pedagógicas de los docentes de práctica clínica del pregrado en Enfermería, relacionados con la planeación pedagógica y didáctica, el uso de estrategias y técnicas de enseñanza y el logro de objetivos curriculares. Esto marca retos académicos y profesionales, si bien la disciplina enmarca el actuar del profesional de enfermería, el humanismo y las relaciones interpersonales también tienen que reforzarse en el proceso de enseñanza, para lograr una educación integral de los futuros profesionales de enfermería.

Ante este escenario, surgen las interrogantes: ¿Cómo se está enseñando el cuidado a los estudiantes de enfermería? y ¿Qué propuestas se pueden obtener para mejorar la enseñanza del cuidado?; para dar respuesta a estos planteamientos, las investigadoras de este estudio planearon y llevaron a cabo un curso de capacitación para docentes de la práctica clínica en enfermería, sobre la "Enseñanza del cuidado", con la finalidad de concientizar sobre sus prácticas pedagógicas y llevar sus estrategias de mejora o solución al contexto real de la práctica docente.

Por consiguiente, el objetivo de este estudio fue intervenir participativamente en la capacitación de profesores para la enseñanza del cuidado, mediante la concientización e interpretación de sus prácticas pedagógicas que trasciendan en el desempeño académico de los estudiantes de la Licenciatura en Enfermería.

\section{METODOLOGÍA}

Esta investigación tiene un enfoque cualitativo-interpretativo, para lo cual se utilizó el método de Investigación-Acción en el área educativa de enfermería, toda vez que 
vincula de manera inclusiva la participación de la comunidad (investigadoras y docentes) con la finalidad de concientizar y mejorar su praxis docente ${ }^{(6)}$.

El desarrollo de esta investigación considera como escenario una institución universitaria de enfermería en el Estado de México. Se solicitó el permiso de las autoridades para poder trabajar con los docentes de práctica clínica que imparten unidades de aprendizaje en el pregrado. Cabe mencionar que la investigación se deriva de un macroproyecto titulado "Prácticas pedagógicas para un modelo de enseñanza innovador del cuidado en enfermería - Brasil y México", aprobado por la Comisión de Ética del Organismo Académico.

Las etapas que siguió esta investigación están basadas en el Modelo de Investigación-acción de Susman y Evered ${ }^{(7)}$.

\section{Diagnóstico}

Derivado de los resultados del macroproyecto antes citado, se concluyó que los docentes de la Facultad de Enfermería definen las prácticas pedagógicas como: los procesos que explican la forma de mantener una interacción más fuerte con el alumno y que ésta, al final, se refleja en un aprendizaje más significativo, por lo que ubican al alumno como un agente activo y responsable en la secuencia de aprendizaje. Si bien este concepto tiene coherencia con la definición de prácticas pedagógicas, se identificaron ciertas dificultades y desafíos al hablar sobre la praxis de las mismas, ya que los docentes mencionan que muchas veces la relación con los alumnos se dificulta por factores como la planeación pedagógica y didáctica, el uso de estrategias y técnicas de enseñanza, el logro de objetivos curriculares y la comunicación, como se evidencia con los siguientes argumentos:

"[...] Creo que tenemos cierto miedo, porque... bueno, aprenderán lo que les enseñamos, así que, si no sabemos, no podemos enseñar [...] También tuve que aprender a organizarme para expresarme frente al grupo [...] Todo esto en algún momento es difícil para nosotros, pero luego nos vamos [...] adquiriendo más la forma en que nos organizamos, para que también sea más fácil para ellos y para nosotros" E2.

"En un principio me era difícil enseñar a los estudiantes, cómo lograr los objetivos del programa, sabía que me tenía que documentar, actualizar y mover, aprender e implementar técnicas de enseñanza" E3.

[...] mis dos dificultades: Primero, el contenido que es muy amplio en comparación con el tiempo y el otro es que, con grupos muy grandes, es difícil para mí evaluar las habilidades [...] La verdad es que intento hacerlo [usar metodologías activas], pero es bastante largo y, en cierta medida, es mucho más trabajo para el maestro, [...] E4.

"Dado que cada estudiante aprende de una manera diferente, creo que lo más complicado es tener diferentes métodos de aprendizaje y conocer a los estudiantes a medida que aprenden, porque solo puedo tener un método, pero no sería efectivo para uno, todos tienen... diferentes habilidades de aprendizaje E5. 
"En enfermería nos falta esa formación pedagógica y estrategia...tenemos el conocimiento, lo sabemos, pero nos falta mucho poder compartirlo con los alumnos de manera más acertada" ... E6.

Consecuentemente, bajo la reflexión de las vivencias y experiencias cotidianas producto de la práctica docente en enfermería, las investigadoras tomaron la decisión de intervenir activamente en la mejora de estas situaciones mediante la realización de un curso cuya finalidad sería concientizar y mejorar sus prácticas pedagógicas en la enseñanza del cuidado.

\section{Planificación de la acción}

La intervención-acción de las investigadoras hacia los docentes de práctica se realizó mediante un curso-taller con dos convocatorias, una para el turno matutino y otra para vespertino. Se trabajó con 30 profesores de pregrado de Enfermería seleccionados a conveniencia de las investigadoras, los cuales cumplieron los criterios de: Mínimo 3 años de experiencia en la docencia, impartición de asignaturas prácticas clínicas o comunitarias en el programa de pregrado en enfermería e inscripción a los cursos ofertados.

El curso se programó con duración de una semana, cinco horas diarias, 25 horas en total. Las temáticas abordadas en esta intervención fueron: epistemología del cuidado, cuidado humanizado, pensamiento reflexivo y crítico, modelo educativo de la licenciatura, enseñanza y prácticas pedagógicas en enfermería, mismas que fueron impartidas por docentes de tiempo completo e investigadoras de la institución educativa.

Cabe mencionar que para el desarrollo de las sesiones del curso se utilizó el enfoque teórico propuesto por el Arco de Maguerez en sus cinco etapas: 1) Observación de la realidad, 2) Puntos clave, 3) Teorización, 4) Hipótesis de solución, 5) Aplicación a la realidad.

\section{Realización de la acción}

Durante cada sesión del curso se documentaron las experiencias, opiniones y perspectivas de los participantes mediante audios, previo consentimiento. Para esta etapa se fomentó la reflexión y crítica de los profesores asistentes al curso, con el objetivo de que grupalmente se identificaran las características, problemas y posibles soluciones respecto a su enseñanza y prácticas pedagógicas. Se utilizó el análisis de contenido para clasificar e interpretar los testimonios de cada participante y se recurrió a la categorización abierta, la que dio como resultado cuatro categorías y subcategorías: 


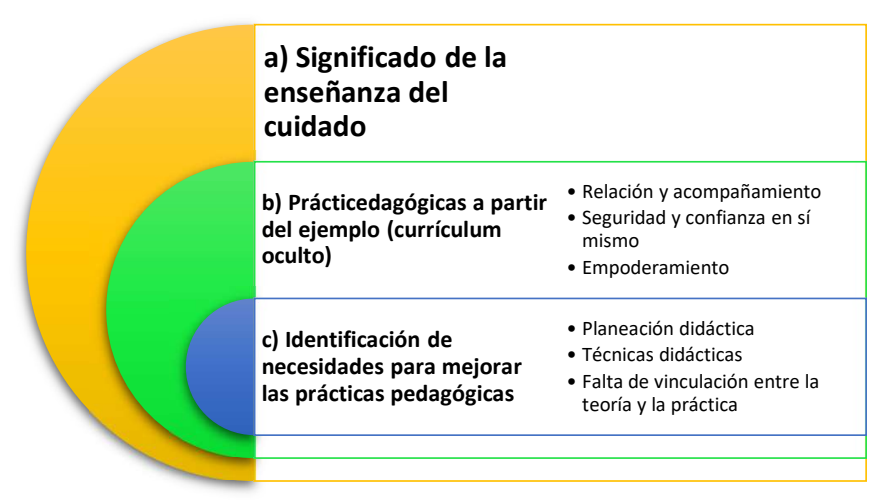

Fuente: Creación propia, 2021.

Para preservar la identidad de los participantes, sus declaraciones se identificaron con códigos formados por la letra "P" (participante) seguido del número correspondiente que se les asignó durante la realización de los encuentros.

\section{Evaluación}

La evaluación del curso se desarrolló mediante una valoración cualitativa, en la que se obtuvieron comentarios y conclusiones finales por parte de los docentes participantes

\section{Definición de nuevos conocimientos}

Como resultado de la acción investigativa, se observaron cambios en las actitudes y disposición de los docentes para mejorar su práctica docente, adquiriendo compromisos individuales y colectivos para una enseñanza de calidad en enfermería.

\section{RESULTADOS}

Los resultados que se muestran a continuación representan a las tres últimas etapas del Modelo de Investigación-acción de Susman y Evered: realización de la acción, evaluación y definición de nuevos conocimientos.

\section{Realización de la Acción}

En esta etapa se documentaron las experiencias y opiniones de los docentes frente a la realidad sentida y vivida en su práctica docente de manera cotidiana, entre este cúmulo de reflexiones se puede identificar a) Significado de la enseñanza del cuidado, la cual consideran que es una construcción de una relación humana, con el empleo de estrategias para fortalecer las competencias del alumno:

Enseño para trascender en vida, para un bienestar personal y social. P-2

Con la finalidad de fortalecer conocimientos, habilidades y actitudes para la vida cotidiana -formación de personas-. P-8

Acrecentar el conocimiento de uno mismo. P-9 
Mejorar las estrategias de aprendizaje y conocimiento de la persona a partir de las experiencias que el docente tuvo como alumno. P-10

También, entre los discursos se identificaron ideas y concepciones alrededor de la aplicación del cuidado, en donde la enseñanza del cuidado inicia con el autocuidado que infunden los docentes en cada uno de los estudiantes, como se observa en el siguiente relato:

Concientizar a los estudiantes a brindar un cuidado humano o humanístico, yo me preocupo como docente por sus necesidades fisiológicas o las necesidades que él tenga, entonces él se va a preocupar por las necesidades de su paciente; porque sabe que es una acción que se debe tener como profesional de enfermería, a diferencia, de como dicen algunos docentes: no puedes comer, te tienes que levantar a las 5 de la mañana para que desayunes, o sea no puedes venir a desayunar aquí ¿no? Entonces él dice: no pues si ella no me deja desayunar porque tengo que dejar desayunar a otras personas y vuelve a ser como el ejemplo. P-17

Conforme las sesiones de intervención trascurrían, los docentes autorreflexionan y pueden expresar la forma en que las enseñanzas obtienen un mayor impacto cuando parten del ejemplo, por ello se describen sus experiencias en las b) Prácticas pedagógicas a partir del ejemplo (currículo oculto):

... también con nuestro ejemplo, o sea, si yo puedo, tú también puedes, y se va haciendo como una cadenita, si mi maestro puede, yo también puedo, y esa palabra es muy grande, muy fuerte, con un gran significado y a la vez es muy sencillo; porque son cosas quizá no son fáciles pero sí sencillas, que nosotros tenemos la capacidad de hacer y enseñarle al alumno, que poco a poco con esas acciones en las que los vamos formando, lo que vamos aprendiendo, pues podemos hacer grandes trabajos, al final del día sí es creer que somos capaces, obviamente es echar la cadenita a ellos para que el fuego de su conocimiento se encienda. $P-9$

Derivado es estas reflexiones, los docentes también consideran que establecer una relación y acompañamiento durante las prácticas clínicas, genera una interacción docente-estudiante, que permite fortalecer el sentido de pertenencia a la profesión de Enfermería, como lo muestran los siguientes comentarios:

Yo creo que el que el alumno se identifique con la carrera es de raíz, es una situación muy importante, si desde primero no está consciente de la responsabilidad social y el impacto que tiene su carrera, no se identifica con la misma, ahora el impacto que tiene ser un enfermero o una licenciada en enfermería...retomar desde la Universidad que conlleva tener valores, tener compromiso y responsabilidad social, toda esa situación conlleva a que el alumno se sienta identificado con su Universidad. P-12

Últimamente, para conocer de alguna manera a los alumnos hice una ficha de identificación, quiénes son, a qué se dedica en su tiempo libre, si le gusta enfermería, por qué estudió enfermería y, me he dado cuenta que realmente 
hay alumnos que es su última opción o están estudiando enfermería, porque así lo quiso la familia, entonces durante el desarrollo de la práctica, tal vez, el apoyo y la motivación son cualidades para ser enfermero y hacen que les guste, que ellos vean la esencia de lo qué es ser enfermero, sin embargo, también a veces se enfrentan con el personal que realmente está igual, por ejemplo, hablando emocionalmente, la verdad es que tenemos personal de enfermería en los hospitales que están así, o sea que llegaron a ser enfermeros por que fue su última opción...P-4

También conoces muchos problemas o muchas situaciones, ejemplo, están aquí sosteniendo su carrera, no tienen dinero a veces para el pasaje, yo he visto que de esa manera también impacta, o sea son muchas situaciones, pero si es escucharlos y motivarlos para descubrir esas cualidades o habilidades que ellos tienen, eso es bueno, por mi parte en lo personal he aprendido también de los alumnos. P-6

Sumado a esta reflexión, los docentes consideran que sin duda el fomento de la seguridad y confianza en sí mismo, es una fortaleza que les ayuda a los estudiantes a enfrentarse a situaciones adversas, que los preparan para su vida profesional.

Yo los apoyo, les brindo seguridad, siempre les digo -tú puedes-. Bueno, yo como docente le brindo seguridad y motivación al alumno, para que siga estudiando, tiene el conocimiento, disposición e iniciativa, entonces ahí en la misma institución aprovecharlo. P-10

Más que nada sería convencerlos de que tengan esa confianza en ellos, porque en primer semestre se les da anatomía, muchas materias que contribuyen a su formación, pero nunca se les habla de que ellos deben de creerse enfermeros, desde el primer momento que entran aquí a la Facultad y dicen soy alumno de la Facultad de Enfermería, pero lo dicen como cabizbajo, así como que no se lo creen; y la forma en que les hablan, les dicen alumno o joven, entonces, sí es necesario que se lo crean, veo que no se les dice el enfermero. P-11

...todos estamos enseñando de diferente manera, yo creo que debemos de enseñarnos, somos colegas; yo le digo al alumno te voy a enseñar, si se puede con ayuda del enfermero, para hacer un procedimiento, no me pongo en el plan digamos de docente, si no en el plan de enfermero; le da cierta seguridad y confianza, si yo me voy en el plan de docente el alumno forma una barrera. P-1

En este sentido, al analizar sus experiencias como docentes, ellos consideran que el empoderamiento debe trabajarse con los estudiantes, toda vez que han identificado la vinculación entre la identidad profesional y el ser profesionistas de enfermería, como una manera en que los alumnos puedan desarrollar todas sus capacidades:

Pero al final somos enfermeros, para tener esa identidad profesional qué es lo que tienes que hacer...tienes que ser profesional, que si hay cosas que todavía les faltan o algunas como ciertas actitudes...entonces pues ahí vamos a fortalecer la identidad y también yo veo que el alumno debe de aprender un objetivo que es el de cuidar, cómo lo vas a cuidar, el estar ahí y decirle te estoy 
ayudando, yo a mis alumnos les digo vamos a enseñarte como enfermero...estar como profesional. P-17

Probablemente lo que demandamos los docentes, es hablarles de que ellos se la tienen que creer, porque en otras carreras ya son el químico, dentista, odontólogo, en todas las materias les dicen así, si no simplemente decirle, oye si estás seguro de querer estudiar, entonces ya comiénzate a creer enfermero, te falta mucho conocimiento, sí claro, pero comienza a creer que tú lo vas a hacer y vas a ser el mejor, pregúntale a un alumno de sexto y lo juntas con otros alumnos ¿Qué estudias? , porque todavía no son capaces de decir con orgullo Enfermería, les falta eso. P-11

Además, consideran que el empoderamiento les permite despeñarse en diferentes contextos de la enfermería, sobre todo, en aquellos que exigen una autonomía, liderazgo y toma de decisiones, así como exigen la relación con grupos multidisciplinarios, donde los estudiantes deben posicionarse como expertos ante el cuidado de la salud.

En el campo de salud pública, al estar en la comunidad es muy diferente, porque el alumno ahí lo es todo, el alumno ahí es el que sabe, el que atiende, el que da todo y las personas que están a su alrededor lo empoderan mucho más, porque dicen cómo es posible que usted sepa tanto, cómo es posible que siendo enfermero usted sepa todo eso, -yo pensé que solo canalizaban, -yo solo pensé que inyectaba y canalizaba-; y ahí se cambia el paradigma, pero dentro del hospital el alumno tiene muchos agentes que lesionan su autoestima como profesional $P-13$

...ciertos alumnos que he tenido en la práctica de salud ocupacional, tienden a estar involucrados con otros personajes que no tienen nada que ver con la salud, por ejemplo: ingenieros, inclusive hasta el presidente de la empresa, entonces eso le da al alumno otra oportunidad de poder relacionarse con otros individuos o profesionistas que no son del área de la salud y entonces como él es el único que tiene la posibilidad de expresar ese poder en su área, tiene que empoderarse y decir, tengo que fomentar la salud con ellos y entonces ellos ven que sí tiene el compromiso y que sí son una figura de autoridad ahí, que tienen voz y voto. $P-12$

Estos testimonios son evidencia del ejercicio de reflexión que hacen los docentes sobre sus prácticas pedagógicas, producto de las temáticas abordadas en el curso.

En las últimas sesiones y producto de un autoanálisis sobre sus prácticas pedagógicas, los docentes han llegado a la c) Identificación de necesidades para mejorar las prácticas pedagógicas, en esta categoría los profesores identificaron que la planeación didáctica que guía algunas unidades de aprendizaje presenta ciertas debilidades, que ellos describen:

Nosotros necesitamos ver realmente el programa de la asignatura, tanto teórica como práctica, para reunir los elementos y recursos necesarios para lograr las competencias $y$, quizá tengamos que empezar en la parte formal e ir mejorando la parte real de la práctica... P-3 
En el desarrollo de la práctica se busca que los alumnos adquieran habilidades, pero, las clases teóricas a veces aportan poco y, lo que ya había planeado, no aplica porque los estudiantes tienen deficiencias, entonces esos planes a veces no se pueden aplicar al cien, ejemplo: los campos clínicos a veces son reducidos, entonces los alumnos están 5 a 6 días en un área y ahí no se logran alcanzar habilidades, a veces lo planeado no es aplicable a la realidad P-8.

Siguiendo con ese ejercicio autorreflexivo, algunos docentes aceptaron tener debilidades ante la aplicación de las técnicas didácticas:

Yo pienso que me faltan técnicas, es decir, lo hago de manera empírica, yo quisiera tener esa parte fundamental de cómo puedo fortalecer el aprendizaje del alumno. P-1

Pienso que las estrategias o técnicas de enseñanza, a veces nos fallan, pero, se requiere de valor para reconocer que como docentes se tienen fallas en la enseñanza, que las maneras de hacer no siempre son las mejores... P-11

... ¿Cuál es la estrategia que vamos a hacer para trabajar la parte procedimental y la parte actitudinal? Entonces a lo mejor un aprendizaje basado en problemas...yo no le voy a decir a los alumnos los conceptos, porque eso lo pueden bajar de Internet, lo va a copiar y no lo va a leer, lo que voy a hacer en mi enseñanza, es que cada contenido esté centrado en la realidad, donde el alumno tenga que aplicar...el conocimiento, la combinación, el análisis, la síntesis y la evaluación y de esa manera, quizás pudiéramos llegar al otro lado y haber cruzado ya la zanja, estamos así del otro lado P-15.

En adición a estas experiencias sentidas por los profesores, reconocen que en su ejercicio docente se enfrentan a la falta de vinculación entre la teoría-práctica, como se describe a continuación:

Yo creo que nosotros en nuestra práctica docente, en el escenario áulico o clínico, ya sea comunitario u hospitalario ¿Qué es lo que debemos enseñar en el alumno? O sea, vamos a hacer énfasis sobre la parte cognoscitiva o en la parte declarativa y, a lo mejor ahí donde nos hemos quedado, si lo vemos por la taxonomía de Bloom, puro conocimiento, puro conocimiento y el alumno que nos recite todo, está bien, pero no hemos pasado al siguiente escalón, que es la comprensión, entonces nos estamos quedando en esa parte declarativa. P14

Surge la necesidad de sentar a los colegas a integrar la teoría y la práctica y que hagan los dos el mismo programa, como si el de práctica se fuera ir a teoría y como si el de teoría se fuera a práctica, si ahorita no podemos, no podemos exigir que hoy me den teoría y el siguiente semestre la práctica, pues por lo menos sentarnos para que los dos comulguen o dialoguen, pero que realmente, a lo mejor el tiempo no nos da y a lo mejor solo podemos cambiar en la unidad uno, pero que con esa sea suficiente, que nos dé tiempo de poder cambiar las cuatro unidades o las cinco unidades pero, que llevemos esa parte para que nosotros podamos checar que tanto sí, que tanto podemos promover este tipo de aprendizaje participativo para ellos. P-4 
Sin duda las experiencias de los docentes permiten describir las inquietudes y preocupaciones sentidas durante su práctica cotidiana y desde un punto de vista crítico, parece que ellos presentan dudas sobre la práctica de los procesos de enseñanza necesarios en los escenarios prácticos; los argumentos recopilados con respecto a sus comentarios del curso aplicado se presentan a continuación.

\section{Evaluación}

En esta etapa los docentes expresan principalmente sus agradecimientos hacia los expertos que impartieron el curso, a las autoridades de la institución por preocuparse por sus experiencias e incertidumbres frente a su ejercicio docente:

Yo les agradezco que se tenga ese interés con nosotros porque, no en gestiones atrás realmente no nos tomaban como que aquí te toca trabajar esto y quien sabe cómo lo hagas y ese es el interés que tiene la facultad por mantenernos capacitados y actualizados para el alumno y de ahí es como se agarra uno también de eso ¿no? Porque si no yo no tengo la menor idea de cómo transmitirlo ¿Cómo se lo voy a enseñar a mi alumno no? Porque hay exigencias, pero no hay esa parte de capacitación y yo si le agradezco Doctora que siempre se ha tenido esa inquietud de nosotros estarnos capacitando y estos cursos en el Inter yo ya los extrañaba $P-2$

Estos comentarios dejan en evidencia la necesidad sentida de los docentes por un acompañamiento y guía académica-profesional, así como de una capacitación continua para mejorar y actualizar sus prácticas pedagógicas.

\section{Definición de nuevos conocimientos}

Ésta es la última etapa de la investigación-acción y es donde se describen los aprendizajes resultados de la intervención, por lo que es importante destacar que el ejercicio de reflexión grupal sobre su desempeño en el ejercicio docente les permitió a los docentes asumir compromisos consigo mismos, así como fortalecer los equipos de trabajo para lograr mejores resultados de aprendizaje con los estudiantes, como se describe a continuación:

... el enriquecernos con todas nuestras opiniones, con nuestras vivencias...fue un curso demasiado rico y claro como todo ¿no? A lo mejor faltó tiempo o nos faltó a nosotros ser más dinámicos, pero considero que fue un buen curso y sobre todo el aprendizaje que tuve de nuestro trabajo en equipo como gremio, como compañeros de práctica fue muy bueno, gracias. P-5

...quiero decirles que veo la gran diferencia que hubo entre el año pasado y este...Bueno ahora voy a estar al frente de un grupo...me sentía arropada por algunos de los compañeros que me decían: tú no te preocupes, mira no te va a costar trabajo, tienes que hacer esto, tienes que hacer lo otro. El día de hoy me siento sumamente feliz y muy motivada, justamente porque las veo que están ustedes comprometidas, porque están al frente, porque son caras conocidas, porque nos están infundiendo toda esta confianza, porque nos están hablando del empoderamiento que nos hace falta afuera, porque veo todas las circunstancias y me doy cuenta de que todos mis compañeros cursan por las 
mismas problemáticas que nosotros cursamos, porque esta convivencia nos fortalece como profesionales $P$-7

Sumado al trabajo en equipo, los profesores consideran fundamental continuar con la capacitación continua:

como las compartimos aquí en el grupo, en los varios equipos que conformamos en el transcurso del curso y conforme fueron pasando los días, yo me di cuenta que hay cosas en las que coincidimos, en opiniones, en técnicas y en qué hacemos bien, solo lo que falta es fortalecer, seguirnos capacitando y considero que esa es una buena oportunidad para conocernos, para compartir, para entender el punto de vista del compañero, para que quizá nos entienda a nosotros y nos fortalezca con eso que saben, bueno los temas que ustedes nos dieron la verdad es que son imuy buenos!, o sea, nos enriquecen muchísimo. P-5

Por último, los docentes reconocen las oportunidades de mejora que ellos pueden tener en sus prácticas pedagógicas:

Yo creía que había situaciones que solamente en mi práctica se vivían, que a lo mejor como docente sólo yo percibía ciertas cosas de mis alumnos, pero en este curso me di cuenta que no, que hay situaciones generalizadas, así como hay alumnos que tienen muchas ganas hay alumnos que debemos de apoyar...Pero, el enriquecernos con todas nuestras opiniones, con nuestras vivencias...fue un curso demasiado rico...y sobre todo el aprendizaje que tuve de nuestro trabajo en equipo como gremio, como compañeros de práctica fue muy bueno, gracias. P-5

... fue realmente un honor...he aprendido mucho...uno se va con el compromiso y con el humanismo...así mismo para mí siempre la palabra mágica es y será trascender, trascender para y con los alumnos...alguien me decía ¿tienes hijos?, no, no tengo hijos, tengo muchísimos hijos, muchísimas plantitas, semillitas en las cuales se insertó algo de mí y ahorita están germinando, están siendo mis colegas...P-4

... me voy con muchas expectativas, tengo que seguir aprendiendo y tengo que seguir documentándome para poder resolver situaciones que se presentan en la práctica y mi objetivo también es dejar esa semillita...me he dado cuenta que sí, o sea he dejado ahí un granito, aunque sea un granito de mostaza como dice $y$ he visto a mis alumnos, salir adelante. Entonces me voy contenta $y$ sobre todo con ese reto porque sé que todavía me faltan muchas cosas por aprender, gracias... P-8.

\section{DISCUSIÓN}

Cabe hacer mención que este estudio contempló la reflexión y compromiso profesional de los docentes de Enfermería en la enseñanza del cuidado a los estudiantes, a su vez, permitió compartir puntos de vista divergentes y convergentes que se unen en un objetivo común: formar a seres humanos que cuidan personas. Lo cual coincide con la propuesta de proyectos educativos de gran alcance para la formación de sujetos responsables y comprometidos en intervenciones creativas 
sobre la realidad, destacando que esto conlleva un gran desafío. Las prácticas pedagógicas son una construcción compartida de nuevos compromisos éticos y políticos para la producción en salud, para la enseñanza y el aprendizaje, para la investigación científica ${ }^{(8)}$.

De la misma forma, se encontraron dificultades o situaciones que complejizan la enseñanza del cuidado, derivadas de la interrelación alumno-docente y de los diversos ambientes de aprendizaje en la práctica de Enfermería; con respecto a esto, Barbel, refiere que la concepción de la educación histórico-crítico, tiene un mayor propósito de preparar al estudiante o ser humano en tomar conciencia de su mundo y actuar intencionalmente para transformarlo ${ }^{(9)}$, siendo esa transformación la forma de trascender de los docentes en los alumnos.

En la formación de profesionales de enfermería, entre el 40 al $50 \%$ de créditos, son de práctica clínica y las metodologías de enseñanza mayor recomendadas que refuerzan la vivencia son el aprendizaje basado en problemas (ABP), la práctica basada en la evidencia (PBE), el estudio de casos, la simulación clínica, la autorización y la supervisión clínica. (10) así como laboratorios vivenciales y socio dramas (11). Esto tiene relación con la forma en que los profesores brindan la enseñanza del cuidado a los estudiantes de la Facultad.

Las prácticas pedagógicas constituyen un proceso que se despliega en el contexto del aula, laboratorio o campo de práctica, en los que se pone de manifiesto una determinada relación docente-estudiante centrado en la construcción y aplicación del conocimiento, involucran un entramado de interrelaciones y transrelaciones con aprendizajes compartidos (11), es en este mismo sentido que los docentes de la Facultad definen como componentes indispensables de sus prácticas pedagógicas, la relación y acompañamiento a los alumnos, que favorezca el empoderamiento, la seguridad y confianza de sus alumnos.

También se resalta la necesidad de la capacitación continua, como una forma de intercambiar experiencias; resultados que coinciden con los encontrados por Tejada y colaboradores, quienes reconocen la necesidad de capacitación en tecnologías de la información, pedagogía y didáctica que permitan la innovación y aplicación de diversas metodologías de enseñanza, que se constituyen en las herramientas necesarias para lograr el perfil del docente universitario y cumplir con el perfil de egreso ${ }^{(3)}$.

Si bien aún son diversos los retos que afrontar para mejorar las prácticas pedagógicas de los docentes del pregrado en Enfermería, se pudo observar que mediante el curso sobre enseñanza del cuidado se promovió y rescató la visión ética y el humanismo que demanda nuestra profesión y, por ende, la educación de los futuros profesionistas de Enfermería.

\section{CONCLUSIÓN}

La finalidad u objetivo que tienen las prácticas pedagógicas de los docentes de la Facultad es para: Trascender, disminuir miedos en el alumno, lograr y desarrollar competencias profesionales y humanas y acrecentar conocimientos. 
Las prácticas pedagógicas se deben enseñar a partir del ejemplo, o lo que conocemos como el currículo oculto.

La relación y acompañamiento a los alumnos, el empoderamiento, la seguridad y confianza en sí mismos, son prácticas pedagógicas que deberían prevalecer en la enseñanza del cuidado de los futuros Licenciados en Enfermería.

Entre los docentes participantes se observaron necesidades pedagógicas y didácticas para favorecer la enseñanza, así como una falta de vinculación entre la teoría y la práctica.

Se requiere fortalecer los equipos de trabajo y la capacitación continua en la enseñanza del cuidado, distinguiendo que existen oportunidades de mejora en el desarrollo de las prácticas pedagógicas.

\section{REFERENCIAS}

1. Zambrano Santos, Roberth Olmedo; Macías Alvia, Aida Monserrate; Solórzano Solórzano, Sidar Edgardo, Fundamento pedagógico del cuidado humano en la práctica de enfermería Revista Digital de Postgrado, vol. 8, núm. 3, 2019. Universidad Central de Venezuela, Venezuela. [citado 2021 Mar 20] Recuperado de: http://portal.amelica.org/ameli/jatsRepo/101/101778008/101778008.pdf

2. Báez-Hernández, Francisco Javier, Nava-Navarro, Vianet, Ramos-Cedeño, Leticia, Medina-López, Ofelia M., El significado de cuidado en la práctica profesional de enfermería. Aquichan [Internet]. 2009;9(2):127-134. Recuperado de: https://www.redalyc.org/articulo.oa?id=74112142002

3. Tejada S., Ramirez E.J., Díaz R.J., Huyhua S.C. Práctica pedagógica en la formación para el cuidado de enfermería. Enferm. univ [revista en la Internet]. 2019 Mar [citado 2021 Mar 19]; 16( 1 ): 41-51. Disponible en: https://doi.org/10.22201/eneo.23958421e.2019.1.577

4. Medina González I, Tafur Castillo J. Didáctica de la Enfermería, análisis desde sus componentes en el contexto actual. Educación Médica Superior [Internet]. 2018 [citado 20 Mar 2021]; 32(4): [aprox. 0 p.]. Disponible en: http://www.ems.sld.cu/index.php/ems/article/view/1603

5. Gómez, Olga Janeth, Carrillo, Gloria Mabel, Cárdenas, Diana Carolina, Estrategias para el Aprendizaje Significativo del Cuidado Humano en Enfermería. Archivos de Medicina (Col) [Internet]. [citado 2021 Mar 20]; 2017;17(2):437-444. Recuperado de: https://www.redalyc.org/articulo.oa?id=273854673024

6. Moreno J, Espada, M A. Investigación acción participativa. Revista Perspectivas Sociales. [Internet]. 2013. [noviembre 2020]; 15. http://ineditviable.blogspot.com/iapinvestigacion-accion-participativa.html.

7. Abad Corpa, Eva; Delgado Hito, Pilar and Cabrero Garcia, Julio. La investigación-acción-participativa: Una forma de investigar en la práctica enfermera. Invest. educ. enferm[online]. 2010, vol.28, n.3, pp.464-474. ISSN 0120-5307. http://www.scielo.org.co/scielo.php?pid=S0120-

$53072010000300017 \&$ script $=$ sci_abstract\&tlng=es

8. Donato, LBG., Alvarez, AM., Santos, LMW. La educación de enfermería: calidad innovación y responsabilidad. Rev. Bra. Enfermagem. [Internet].2014 [citado 2021 Ene 26]; 64 (4): 503-504. https://www.scielo.br/pdf/reben/v67n4/es_0034-7167reben-67-04-0499.pdf 
9. Do Prado, ML., Sobrinho, SH., Velho MB., Espindola, DS., Shubert, BV. El Arco de Chales Maguerez: una reflexión sobre las estrategias de metodología ativa para la transformación de los profesionales del área de la salud. Esc Anna Nery. [Internet]. $2012 . \quad$ [enero 2021];

https://www.scielo.br/pdf/ean/v16n1/v16n1a23.pdf

10. Ruiz-Davila, E. La enseñanza clínica en enfermería. Rev. Mex. Enf. [Internet]. 2015 2021];

3:6-10.

http://www.innsz.mx/imagenes/revistaenfermeria/septiembre-2015.pdf

11. Flórez-Gutiérrez C, Mayorga-Palacios A del P, Vargas-Escobar LM. La práctica formativa como escenario de aprendizaje significativo del cuidado de enfermería. Rev. Colomb. Enferm. [Internet]. 25 de abril de 2017 [citado 25 de marzo de 2021]; 14:93103.

Disponible

en:

https://revistacolombianadeenfermeria.unbosque.edu.co/index.php/RCE/article/view/2 031 